\title{
The Mediating Role of Early Maladaptive Schemas in the Relationship between Childhood Traumatic Events and Complex Posttraumatic Stress Disorder Symptoms in Older Adults (>64 Years)
}

\author{
Eleni Vasilopoulou, Thanos Karatzias, Philip Hyland, Hannah Wallace \& \\ Azucena Guzman
}

To cite this article: Eleni Vasilopoulou, Thanos Karatzias, Philip Hyland, Hannah Wallace \& Azucena Guzman (2020) The Mediating Role of Early Maladaptive Schemas in the Relationship between Childhood Traumatic Events and Complex Posttraumatic Stress Disorder Symptoms in Older Adults (>64 Years), Journal of Loss and Trauma, 25:2, 141-158, DOI: $10.1080 / 15325024.2019 .1661598$

To link to this article: https://doi.org/10.1080/15325024.2019.1661598

Published online: 06 Sep 2019.

Џll Article views: 436

View Crossmark data ¿
Submit your article to this journal $\sqsubset$ 


\title{
The Mediating Role of Early Maladaptive Schemas in the Relationship between Childhood Traumatic Events and Complex Posttraumatic Stress Disorder Symptoms in Older Adults ( $>64$ Years)
}

\author{
Eleni Vasilopoulou ${ }^{a}$, Thanos Karatzias ${ }^{b, c}$ (D) Philip Hyland ${ }^{d}$ (D), \\ Hannah Wallace ${ }^{e}$, and Azucena Guzman ${ }^{\mathrm{f}, \mathrm{g}}$ \\ ${ }^{a}$ Department of Clinical Psychology, University of Edinburgh, Edinburgh, UK; ${ }^{b}$ Mental Health,

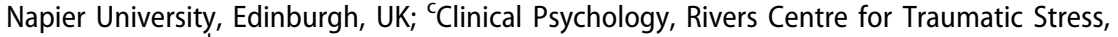 \\ Edinburgh, UK; ${ }^{d}$ Psychology, Maynooth University, Kildare, Ireland; ${ }^{\circ}$ Clinical Psychology, NHS

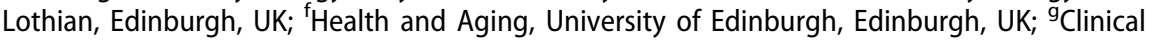 \\ Psychology, Department of Clinical Neuroscience, Western General Hospital, Edinburgh, UK
}

\begin{abstract}
The current study investigated the mediating role of Early Maladaptive Schemas (EMS) in the association between childhood trauma and Complex PTSD (CPTSD) symptom severity in a clinical sample of 42 older adults ( $>64$ years). It was found that EMS total score mediated the relationship between childhood trauma and CPTSD symptom severity. Two second order schema factors (Disconnection; Impaired Autonomy) also had a mediating role in this relationship. Results provide preliminary support for the utility of interventions targeting EMS, particularly in the domains of Disconnection and Impaired Autonomy, in order to alleviate CPTSD symptoms. Future research is required to replicate these results within larger samples and to examine the efficacy of schema and cognitive interventions within trauma exposed older adults.
\end{abstract}

\section{ARTICLE HISTORY}

Received 27 June 2019

Accepted 23 August 2019

\section{KEYWORDS}

childhood trauma; schemas; CPTSD; older adults

\section{Introduction}

Complex Posttraumatic Stress Disorder (CPTSD) has recently been included in the 11th Revision of the International Classification of Diseases (ICD-11) as a sibling condition to Posttraumatic Stress Disorder (PTSD) (World Health Organisation, 2018). In contrast to PTSD which can be triggered by a single event, CPTSD typically, although not always, results from exposure to cumulative trauma, particularly during childhood (Cloitre, Garvert, Brewin, Bryant, \& Maercker, 2013; Hyland et al., 2017; Karatzias et al., 2017b). CPTSD symptoms reflect disturbances in self-organization (affective dysregulation, negative self-concept and disturbances in 
relationships) in addition to the three PTSD symptom clusters (reeexperiencing, avoidance/numbing, and sense of threat).

Lifetime population prevalence of CPTSD is estimated around 3.3\% (Karatzias et al., 2017a), slightly lower than that typically reported for PTSD (4\%), CPTSD, however, constitutes a more common disorder among clinical samples (Karatzias et al., 2017a). In addition, due to the greater diversity of symptoms, CPTSD can have a more pervasive impact on an individual's life and has been associated with more severe functional impairment compared to PTSD (Hyland et al., 2017; Karatzias et al., 2017b). The high clinical occurrence and debilitating impact of CPTSD emphasize the need for early identification of the disorder and for the development of targeted treatments in order to improve service users' prognosis and functioning.

In recent years, there has been a rise in publications on the presentation and correlates of PTSD symptoms amongst older adults. The need for this is highlighted by an increasing aging population (Dall et al., 2013), as well as by research arguing for unique features of PTSD in the context of later life, particularly with regards to symptom profiles (Goenjian et al., 1994), onset (Hiskey, Luckie, Davies, \& Brewin, 2008) and severity (Parker et al., 2016). In addition, age-associated biological, psychological and social changes may influence PTSD presentation in later life. Decreased health, retirement, interpersonal losses, and financial difficulties more frequently associated with later life, may affect individuals' capacity to adapt to traumatic events (Böttche, Kuwert, \& Knaevelsrud, 2012; Grenade \& Boldy, 2008). The emergence and profile of PTSD in later life may also be influenced by age-related cognitive difficulties (Floyd, Rice, \& Black, 2002; Ruzich, Looi, \& Robertson, 2005).

Despite the recent rise in interest in later life PTSD, the majority of older adult studies have focused on either middle or later life traumatization (Vasilopoulou, Michalska da Rocha, Forsyth, Guzman, \& Karatzias, 2019). Meanwhile, there is a dearth of research on the impact of exposure to childhood trauma and, to our knowledge, no studies have examined the factors affecting CPTSD symptom severity in later life. A better understanding of the factors associated with CPTSD symptoms following early life traumatization is paramount to adequately address the support needs of this population. Research in younger adults has demonstrated the role of Early Maladaptive Schemas (EMS) in predicting the emergence and severity of PTSD following traumatization (Ahmadian, Mirzaee, Omidbeygi, Holsboer-Trachsler, \& Brand, 2015; Price, 2007). EMS are defined as themes regarding oneself and one's relationship with others, which develop during childhood, can have a long-lasting impact, and interfere with how people view themselves and their interaction with others (Young, Klosko, 
\& Weishaar, 2003). Eighteen EMS have been proposed and are subsequently categorized in four secondary schema domains: Disconnection, Impaired Autonomy, Exaggerated Standards, and Impaired Limits (Hoffart et al., 2005). Previous studies have indicated higher EMS among individuals with a PTSD diagnosis compared to controls in a range of populations including veterans (Ahmadian et al., 2015; Cockram, Drummond, \& Lee, 2010) and forensic staff (Boudoukha, Przygodzki-Lionet, \& Hautekeete, 2016). EMS have also been associated with PTSD in adult survivors of interpersonal trauma (Dutra, Callahan, Forman, Mendelsohn, \& Herman, 2008), survivors of childhood sexual abuse (Harding, Burns, \& Jackson, 2012; Karatzias, Jowett, Begley, \& Deas, 2016), and cases of traumatic childbirth (Edworthy, Chasey, \& Williams, 2008). A significant association has also been found between the number of hyperactivated EMS and PTSD symptom severity in individuals seeking treatment for substance misuse (Lecigne \& Tapia, 2018).

Research examining the relative contribution of schema domains in PTSD severity has indicated a stronger association between schemas within the Disconnection and Impaired Autonomy domains and PTSD (Harding et al., 2012; Karatzias et al., 2016; Price, 2007). The Disconnection domain includes social isolation, emotional deprivation, emotional inhibition, mistrust/abuse, and defectiveness schemas. The Impaired Autonomy domain consists of schemas relating to subjugation, dependence, failure, vulnerability, abandonment, enmeshment and insufficient self-control (Hoffart et al, 2005). Previous research suggests that schemas included within these domains may be particularly vulnerable to experiences of trauma and abuse, which could, in turn, explain their stronger association with PTSD (Harding et al., 2012; Lumley \& Harkness, 2007; Young, et al., 2003).

The relationship between EMS and PTSD has been researched in the adult populations (Karatzias et al., 2016), however, no studies have investigated the role of EMS in the development of CPTSD symptoms in older adults ( $>64$ years of age).

Even though CPTSD and PTSD are sibling disorders, CPTSD differs from PTSD in terms of number and nature of symptoms (Elklit, Hyland, \& Shevlin, 2014), the nature of the precipitating trauma exposure (Karatzias et al., 2017b, 2019a), as well as the levels of functional impairment associated with the disorder (Karatzias et al., 2017b). There is currently a lack of evidence regarding the usefulness of existing trauma-focused interventions for CPTSD which are commonly used for PTSD (e.g. CBT or EMDR), while emerging research suggests that CPTSD may require a different therapeutic approach (Karatzias et al., 2019b). Therefore, research into the relationship between EMS and CPTSD might enable the identification of important therapeutic targets for intervention from a theoretical perspective. 
The current study aimed to examine whether EMS mediated the relationship between early traumatic events and CPTSD symptoms in a clinical sample of older adults. The study also sought to investigate the mediating role of second order schema factors in this association. It was hypothesized that EMS would mediate the relationship between childhood trauma and CPTSD severity, and based on previous research (Harding et al., 2012; Karatzias et al., 2016; Price, 2007), it was hypothesized that the second order schema factors of Disconnection and Impaired Autonomy would be significant mediating factors.

A better understanding of the factors associated with the severity of CPTSD symptoms within older adults is important when considering the assessment and early identification of traumatized individuals. Such an understanding could increase our knowledge of how to support and respond to older people who have experienced trauma. In particular, if a positive mediating relationship is established, this study could offer preliminary results on the usefulness of cognitive or schema based interventions with older people. Furthermore, by specifying which schema domains need to be targeted in order to alleviate distress in older people with CPTSD symptoms, this study could guide the focus of future complex trauma interventions within this population.

\section{Method}

\section{Participants}

Participants in this study were individuals who were seen for psychological therapy by psychologists, psychiatrists or community mental health clinicians within the National Health Service (NHS) Older Adult services. Older adults $(>64)$ with a history of childhood trauma (physical abuse, sexual abuse, emotional abuse, physical neglect, emotional neglect) were eligible for participation. In order to participate in the study, service users required to be willing to participate voluntarily and to be able to give written informed consent. Older adults with a diagnosis of Mild Cognitive Impairment or Dementia or who lacked fluency in English were excluded from the study. For ethical reasons and to ensure individuals' safety, this study excluded older adults in crisis, experiencing suicidal thoughts with clear intent to harm themselves. Ethical approval was received from the North of Scotland Research Ethics Service (Ref:17/NS/0117). Recruitment lasted from March 2018 until March 2019.

\section{Procedure}

The researcher contacted Older People's Community Mental Health Teams in Scotland explaining the purpose of the study, study rationale and 
recruitment process. NHS staff were asked to identify potential participants from their caseloads who met inclusion and exclusion criteria. Clinicians were given information sheets to disseminate to identified service users outlining research aims, overview and procedure. Clinicians were asked to request service users' verbal consent for the researcher to contact them in order to provide them with more information about the study. Following a period of at least $24 \mathrm{~h}$, the first author telephoned potential participants who gave their permission to be contacted. If individuals were still interested in taking part, a meeting was arranged to obtain informed consent and to complete study measures. Participants completed all questionnaires with the help of the researcher, who offered clarification when needed.

\section{Measures}

\section{Childhood trauma questionnaire (CTQ, Bernstein \& Fink, 1998)}

Exposure to childhood traumatic events was measured by the CTQ. The CTQ is a 28-item retrospective self-report questionnaire which includes five subscales: Emotional Abuse, Physical Abuse, Sexual Abuse, Emotional Neglect, and Physical Neglect. In addition, the questionnaire includes a Minimization/Denial scale in order to identify the underreporting of traumatic events and to assess recall and reporting biases. Participants are asked to respond to each question on a 5-point scale ranging from "never true" (1) to "very often true" (5). The CTQ has demonstrated strong psychometric properties when used both in clinical and community settings (Bernstein, Ahluvalia, Pogge, \& Handelsman, 1997; Scher, Stein, Asmundson, McCreary, \& Forde, 2001).

\section{Young schema questionnaire - short form, 3rd edition (Young, 2014,}

\section{YSQ-S3)}

The YSQ-S3 is a self-report measure assessing 18 EMS. The items are categorized in four schema factors which replaced Young's previous five schema domains (Young et al., 2003): Disconnection, Impaired Autonomy, Exaggerated Standards, and Impaired Limits (Hoffart, et al., 2005). Participants are asked to rate descriptive statements on a 6-step Likert-scale which ranges from "completely untrue of $m e$ " (1) to "describes me perfectly" (6). The YSQ-S3 provides a total score and higher values are associated with a stronger presence of EMS. This measure has demonstrated good psychometric properties and has shown age neutrality when administered across the lifespan (Pauwels et al., 2014). 
International trauma questionnaire (ITQ) (Cloitre et al., 2018)

The ITQ is a self-report measure of PTSD and CPTSD severity assessing the following symptom: reeexperiencing in the here and now (nightmares and flashbacks), avoidance (internal and external reminders), sense of threat (hypervigilance and hyperarousal), affective dysregulation (hyper- and hypo-activation of emotional responses), negative selfconcept (viewing oneself as a failure and worthless), and disturbances in relationships (avoidance of and difficulties in forming interpersonal relationships). Participants are asked to select on a Likert scale how much a symptom has been bothersome in the past month or how true certain statements are of them, with scores ranging from 0 ("not at all") to 4 ("extremely"). Diagnosis of PTSD requires the endorsement of one of two symptoms from each PTSD cluster, while CPTSD diagnosis requires the endorsement of one of two symptoms from both PTSD and 'Disturbances in Self-organization' clusters. Both diagnoses require the presence of functional impairment. The ITQ is the only validated measure for ICD-11 PTSD and CPTSD.

\section{Results}

\section{Descriptive statistics}

A total of 59 eligible patients were identified by clinicians and were given information about the study during the recruitment period. Of those, 42 agreed to participate (71\%). Participant demographic and population characteristics are summarized in Table 1.

CTQ trauma scores ranged from 30 to 108, with a mean of 62.2 $(S D=15.8)$. Trauma exposure frequencies by type of trauma can be seen in Table 2. Participants reported CPTSD severity scores ranging from 4 to 44 $($ Mean $=23.8, S D=11.7)$. Thirteen participants $(30.9 \%)$ met criteria for a diagnosis of CPTSD, while three participants (7.1\%) met criteria for PTSD. Means and standard deviations of CPTSD symptom clusters can be seen in Table 3. The correlations between childhood traumatic experiences, CPTSD symptoms, EMS, and relevant demographic variables are shown in Table 4.

\section{Analysis}

Descriptive analyses were performed using the Statistical Package for Social Science (SPSS version 23.0). Means and standard deviations (SD) were calculated for continuous variables and frequencies (\%) for categorical variables. Mediation analysis (Hayes, 2013) was implemented to answer the primary research question. Hayes PROCESS macro Model 4 (Hayes, 2013) for SPSS was be used to analyze the data. Bootstrapped 
Table 1. Participant characteristics.

\begin{tabular}{lc}
\hline Variable & M (SD) or $n(\%)$ \\
$n=42$ \\
\hline Age mean (SD) & $71.5(4.6)$ \\
Gender \% & \\
Male & $11(26.2 \%)$ \\
Female & $31(73.8 \%)$ \\
Education mean (SD) & $13(3.6)$ \\
Relationship status & \\
Single & $9(21.4 \%)$ \\
Married & $17(40.5 \%)$ \\
Divorced & $10(23.8 \%)$ \\
Widowed & $6(14.3 \%)$ \\
Employment status & \\
Employed & $4(9.5 \%)$ \\
Retired & $38(90.5 \%)$ \\
Ethnicity & \\
White British & $22(52.4 \%)$ \\
White Scottish & $19(45.2 \%)$ \\
White Other & $1(2.4 \%)$ \\
Income & \\
Less than 14,999 & $17(40.5 \%)$ \\
15,000-19,999 & $8(19.0 \%)$ \\
$20,000-29,000$ & $10(23.8 \%)$ \\
30,000-39,000 & $3(7.1 \%)$ \\
$50,000-59,000$ & $2(4.8 \%)$ \\
$60,000-69,000$ & $2(4.8 \%)$ \\
Medical conditions & \\
Depression & $13(30.9 \%)$ \\
Arthritis & $11(26.1 \%)$ \\
COPD & $10(23.8 \%)$ \\
Cancer & $6(14.2 \%)$ \\
\hline &
\end{tabular}

Table 2. Trauma frequencies.

\begin{tabular}{lc}
\hline Trauma frequencies & $N(\%)$ \\
\hline Emotional abuse & $38(90.5)$ \\
$\quad$ Yes & $4(9.5)$ \\
$\quad$ No & \\
Physical abuse & $26(61.9)$ \\
$\quad$ Yes & $16(38.1)$ \\
$\quad$ No & \\
Sexual abuse & $24(57.1)$ \\
$\quad$ Yes & $18(42.9)$ \\
No & \\
Emotional neglect & $37(78.1)$ \\
Yes & $5(11.9)$ \\
No & \\
Physical neglect & $25(59.5)$ \\
Yes & $17(40.5)$ \\
No & \\
Minimisation/Denial & $32(76.2)$ \\
0 & $9(21.4)$ \\
1 & $1(2.4)$ \\
3 &
\end{tabular}

sampling distribution was used to estimate the indirect effect, the standard error and 95\% confidence intervals for the population value of 'ab' (Preacher \& Hayes, 2004). The CTQ minimizing response bias was entered in the analysis as a covariate, as research has suggested a 
Table 3. CPTSD symptom clusters.

\begin{tabular}{lcc}
\hline CPTSD symptoms & Range & Mean (SD) \\
\hline Reeexperiencing & $0-7$ & $2.67(2.40)$ \\
Avoidance & $0-8$ & $3.81(2.80)$ \\
Sense of threat & $0-8$ & $4.62(2.37)$ \\
Affective dysregulation & $1-8$ & $4.74(2.10)$ \\
Negative self-concept & $0-8$ & $3.79(3.19)$ \\
Disturbances in relationships & $0-8$ & $4.21(2.67)$ \\
PTSD & $1-23$ & $11.10(6.00)$ \\
DSO & $2-24$ & $12.74(6.98)$ \\
CPTSD total score & $4-44$ & $23.83(11.78)$ \\
\hline
\end{tabular}

significant effect of this subscale in the CTQ's discriminative validity (MacDonald et al., 2016).

Sample size calculations were based upon the primary research question. Fritz and MacKinnon (2007) have published guidelines in regard to obtaining an adequate sample size for mediation analysis. The authors proposed a minimum number of participants to achieve 0.8 power depending on the magnitude of the estimated effect sizes for the ' $a$ ' and ' $b$ ' mediation pathways. Previous research (Foa, Ehlers, Clark, Tolin, \& Orsillo, 1999; Karatzias et al., 2016; Wright, Crawford, \& Del Castillo, 2009) has indicated large effect sizes for the ' $a$ ' and ' $b$ ' pathways. Based on these findings, a sample size of 36 was deemed appropriate.

\section{Mediation analyses}

Results indicated that after controlling for minimisation/denial of childhood trauma, EMS total score significantly mediated the relationship between experiences of childhood trauma and CPTSD symptoms $(\beta=.39, S E=.15$, $(95 \% \mathrm{CI}=.08, .67)$. Childhood trauma significantly predicted EMS total score $(B=.6, p=.001)$, which in turn significantly predicted CPTSD symptom severity $(B=.5, p=.001)$. The mediation model accounted for $65 \%$ of the variance in CPTSD symptoms $\left(R^{2}=.65 ; F(3,38)=24.02, p<.001\right)$. In order to estimate the magnitude of the indirect effect, the $k^{2}$ coefficient was calculated based on Preacher and Kelley's (2011) guidelines with results suggesting a large effect size $\left(k^{2}=0.48\right)$.

Three additional exploratory mediation analyses were conducted to examine whether specific second order schemas (Disconnection, Impaired Autonomy, Exaggerated standards) mediated the relationship between childhood trauma and CPTSD severity. Impaired Limits was not entered into the analysis due to the absence of an association in our Bivariate analysis. Of the three second order schema domains, Disconnection and Impaired Autonomy mediated this relationship. The model from childhood trauma to CPTSD severity through Disconnection explained $67 \%$ of the variance in CPTSD scores $(F(3,38)=26.16, p<.001)$ with a significant 


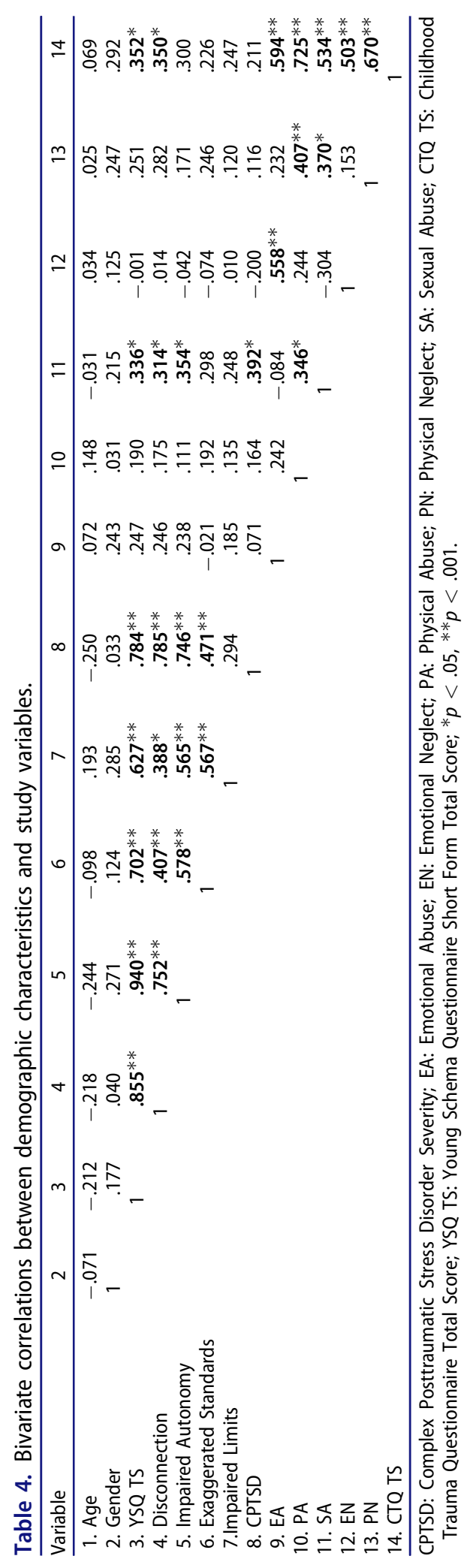


indirect effect $(\beta=.37, S E=.14,95 \% \mathrm{CI}=.09, .63)$. The effect size of the indirect association was strong $\left(k^{2}=0.46\right)$. The model from childhood trauma to CPTSD severity through Impaired Autonomy explained $60 \%$ of the variance in CPTSD scores $(F(3,38)=19.23, p<.001)$ with a significant indirect effect $(\beta=.32, S E=.11,95 \% \mathrm{CI}=.07, .52)$. The effect size of the indirect association was strong $\left(k^{2}=0.38\right)$.

\section{Discussion}

The aim of the current study was to examine the relationship between childhood trauma, EMS, and CPTSD symptoms within an older adult clinical sample. In line with our hypotheses, we found that EMS mediated the association between childhood traumatic experiences and CPTSD symptoms. This effect was due specifically to the second order schema domains of Disconnection and Impaired Autonomy.

Our results provide empirical support for the Schema Therapy model (Young et al., 2003), specifically in regard to its principle that EMS develop in response to adverse childhood experiences. In this study, participants who reported a greater degree of traumatization in childhood were more likely to display elevated levels of EMS. This is not surprising given that early experiences shape expectations regarding the degree of support from one's environment, and can elicit beliefs around the reliability, predictability or emotional availability of others (Pynoos, Steinberg, \& Wraith, 1995). Adverse childhood experiences can disrupt this process and have been shown to have a potentially detrimental impact on how individuals conceptualize themselves and others around them (Estévez, Jauregui, Ozerinjauregi, \& Herrero-Fernández, 2017; Roemmele \& Messman-Moore, 2011). Consistent with previous research (Rezaei, Ghazanfari, \& Rezaee, 2016), our study linked trauma experiences to schemas specifically within the Disconnection and Impaired Autonomy domains. Disconnection schemas include beliefs around others being emotionally unresponsive, hurtful, or manipulative. Participants with elevated Disconnection schemas may see themselves as inadequate, defective and socially isolated. Impaired Autonomy includes schemas relating to abandonment, dependence and vulnerability to harm. People with Impaired Autonomy schemas may have difficulties achieving their goals, separating from others and are more likely to relate to others in a subjugated manner. Such relational patterns are not uncommon among trauma survivors, and can be adaptive, depending on the context (Creech, Benzer, Liebsack, Proctor, \& Taft, 2013). For example, avoidance oriented schemas (e.g. social isolation, subjugation) may be protective in the short term, as they can allow trauma survivors to prevent 
re-traumatization and cope with an unpredictable or stressful environment (Thomson \& Jaque, 2019).

Perhaps, it may feel counterintuitive that the link between EMS and child adversity remained as strong in later life, as that reported in adult or child research (Lumley \& Harkness, 2007; Wright et al., 2009), especially when taking into account gerontological findings on the successful processing of past experiences among older adults (Neupert, Almeida, \& Charles, 2007). A possible explanation for this finding lies in the pervasiveness of EMS: EMS are thought to significantly interfere with the completion of a range of developmental tasks, which may lead to a continuation of negative experiences across the lifespan (Gay, Harding, Jackson, Burns, \& Baker, 2013). Such experiences can provide little opportunity to challenge established maladaptive cognitions and enhance schema rigidity (Atmaca \& Gençöz, 2016; Gay et al., 2013). It is, therefore, not surprising that, within our sample, EMS remained associated with early adverse experiences despite the passage of time.

Furthermore, results from our study supported the relationship between EMS and CPTSD symptoms, by showing that higher levels of EMS (total score) were associated with increased CPTSD severity. Exploratory analyses also demonstrated that the association between childhood trauma and CPTSD severity was mediated by schemas within the Impaired Autonomy and Disconnection domains. These findings are consistent with cognitive models of PTSD which highlight the role of cognitions in the development and maintenance of PTSD symptoms (Ehlers \& Clark, 2000; Foa et al., 1999). Our results are also in line with existing research and guidelines on the treatment of CPTSD which emphasize the importance of challenging unhelpful appraisals in order to promote a positive sense of self and healthier relationships with others (Cloitre et al., 2011; Maercker et al., 2013). Finally, our results are consistent with previous research on the specific contribution of Disconnection and Impaired Autonomy domains to psychopathology following interpersonal trauma (Karatzias et al., 2016).

These findings add to existing research on the link between EMS and psychopathology by showing that EMS constitute a potent vulnerability factor for complex posttraumatic symptoms. These results hold significant implications for the identification of trauma exposed individuals in need of support, as well as for CPTSD symptom prevention and treatment. Considering these outcomes, the inclusion of routine EMS assessment in clinical practice, may be particularly helpful in order to identify trauma exposed service users at risk of developing CPTSD. Similarly, a preventative approach which includes the promotion of positive reappraisal following childhood traumatization, may be beneficial in safeguarding individuals across the lifespan against the development of posttraumatic symptoms. 
More importantly, our results provide preliminary, empirical support for the utility of cognitive-behavioural interventions among traumatized older adults. Specifically, our findings suggest that Schema therapy, with a particular focus on EMS within the Disconnection and Impaired Autonomy domains, may be a useful treatment in order to modify existing core beliefs and reduce CPTSD symptoms within this population. For example, an overt emphasis on themes of safety, trust, and social connectedness may be particularly helpful in challenging Disconnection related schemas. Similarly, an explicit focus on individuals' sources of strength and resilience may help modify Impaired Autonomy related schemas and promote recovery among trauma exposed older adults. These results can inform the very limited research on the efficacy of Schema therapy in later life (Kindynis, Burlacu, Louville, \& Limosin, 2013).

\section{Strengths and limitations}

A number of limitations need to be considered when interpreting the above research findings. First, our study utilized a cross-sectional design therefore we cannot make assertions about causality in the observed relationships. Second, our study included a relatively small sample size which increases the risk for Type I and Type II errors and may prohibit generalization of the above findings. In addition, the majority of our sample comprised of female participants which may further limit the generalisability of findings to the wider trauma population. 'Oldest old' (aged $80+$ ) participants were underrepresented in our sample. Our sample also included a higher proportion of participants meeting criteria for a CPTSD diagnosis compared to those meeting criteria for PTSD. This is in line with research suggesting that CPTSD constitutes a more common disorder among clinical samples (Karatzias et al., 2017a), however it may limit generalisability to PTSD samples. Finally, due to sample size limitations our study did not explore potentially confounding variables, such as adult traumatic exposure, health difficulties and coping mechanisms within our sample.

Notwithstanding these limitations, this is the first study to examine the relationship between EMS and CPTSD within an older adult, clinical sample and provides novel evidence for the role of EMS in CPTSD symptom severity. This study can further our understanding of maladaptive and enduring cognitive schemas in older adults with childhood traumatic experiences and attempts to address a significant gap in research (Videler, van Royen, \& van Alphen, 2012). Further strengths of this study include the robustness and completeness of collected information as the first author supported participants in filling out the study questionnaires. Since our study included a clinical sample receiving support from NHS services, our 
results may be particularly relevant for clinicians working with service users with complex and enduring mental health difficulties.

Future work should focus on the exploration of the causal relationships between childhood trauma, EMS and CPTSD utilizing longitudinal or prospective designs. The inclusion of larger sample sizes will be helpful in exploring the relative contribution of each schema domain in CPTSD severity. Given the sample limitations, future studies would also benefit from the inclusion of 'oldest old', male participants as well as from participants representing different socio-economic and ethnic backgrounds. In addition, given that individuals who have suffered childhood trauma are more likely to be subsequently exposed to traumatic events in adulthood and to develop posttraumatic symptoms (Classen, Palesh, \& Aggarwal, 2005), studies controlling for adult or late life traumatization would be particularly interesting in order to better understand the relative contribution of each trauma type in the development of CPTSD in this population. Further, studies addressing confounding factors affecting posttraumatic symptom severity specific to the older adult population, such as mental and physical health difficulties or maladaptive coping strategies (Vasilopoulou et al., 2019) may also be helpful in further disentangling the relationship between trauma, EMS and CPTSD.

\section{Conclusions}

Our findings suggest that early maladaptive schemas might mediate the relationship between childhood traumatic experiences and CPTSD. Disconnection and Impaired Autonomy domains may play a particularly important role in the onset of CPTSD symptoms. These findings provide support for the theoretical underpinnings of the Schema Therapy model and suggest the potential utility of cognitive interventions in reducing CPTSD severity in older people.

\section{Acknowledgements}

The authors would like to thank Mr Sandy McAfee, former Consultant Clinical Psychologist in NHS Lothian for his support in the initial stages of the research.

\section{Disclosure statement}

The authors declare no conflict of interest.

\section{Funding}

This work was prepared in part fulfillment of the degree of Doctorate in Clinical Psychology, funded by NHS Education for Scotland. 


\section{Notes on contributors}

Eleni Vasilopoulou is a third-year student in the Doctorate in Clinical Psychology at the University of Edinburgh. Research interests include examining factors associated with the development and severity of Posttraumatic Stress Disorders (PTSD) and Complex PTSD. Current research has focused on factors affecting posttraumatic response and presentation in older adults.

Professor Karatzias, is the Director of Research in the School of Health \& Social Care at Edinburgh Napier University, UK and Clinical \& Health Psychologist at the Rivers Center for Traumatic Stress, Edinburgh, UK. He is the former Chair of the British Psychological Society Scotland Working Party for Adult Survivors of Sexual Abuse (BPSSS) and he was a member of the Committee of the British Psychological Society (BPS) Crisis, Disaster \& Trauma Section. In collaboration with national and international research partners he has developed a special interest in the effects and treatment of psychological trauma on physical and mental health; on prison populations; and on people with learning disabilities. The last few years he works in the area of Complex PTSD.

Dr Philip Hyland is a Senior Lecturer at the Department of Psychology, Maynooth University. His primary research area is traumatic stress studies. He is particularly interested in understanding how to most accurately describe and measure psychological responses to trauma; the psychosocial risk factors for different types of traumatic response; and the long-term outcomes of traumatic exposure. Recent research has predominately focused on evaluating the validity of the new descriptions of PTSD and CPTSD in ICD-11. Dr Hyland is the Deputy Statistical Editor of the Journal of Traumatic Stress, and most recently, established the International Trauma Consortium, a global collaboration of researchers and clinicians who wish to advance the science and practice of psychotraumatology.

Dr Hannah Wallace completed her BSc in Applied Psychology at Liverpool John Moores University in 2007 and went on to study for her Doctorate in Clinical Psychology at the University of Edinburgh shortly after this. Dr Wallace was a 4 year specialist aligned trainee with the Lothian Older People's Psychology Service. She graduated in 2014 and has worked in a specialist Older People's Psychology Service in Lothian since. Dr Wallace has specialist interests in complex trauma and neuropsychology.

Dr Azucena Guzman is a Chartered Clinical Psychologist and Lecturer in Health and Ageing at the University of Edinburgh, with 14 years of clinical and research experience in the field of neurodegenerative conditions.

\section{ORCID}

Thanos Karatzias (D) http://orcid.org/0000-0002-3002-0630

Philip Hyland (D) http://orcid.org/0000-0002-9574-7128

\section{References}

Ahmadian, A., Mirzaee, J., Omidbeygi, M., Holsboer-Trachsler, E., \& Brand, S. (2015). Differences in maladaptive schemas between patients suffering from chronic and acute posttraumatic stress disorder and healthy controls. Neuropsychiatric Disease and Treatment, 11, 1677-1684. doi:10.2147/NDT.S85959 
Atmaca, S., \& Gençöz, T. (2016). Exploring revictimization process among Turkish women: The role of early maladaptive schemas on the link between child abuse and partner violence. Child Abuse \& Neglect, 52, 85-93. doi:10.1016/j.chiabu.2016.01.004

Bernstein, D. P., \& Fink, L. (1998). Childhood trauma questionnaire: A retrospective selfreport. San Antonio, TX: Psychological Corporation.

Bernstein, D. P., Ahluvalia, T., Pogge, D., \& Handelsman, L. (1997). Validity of the Childhood Trauma Questionnaire in an adolescent psychiatric population. Journal of the American Academy of Child \& Adolescent Psychiatry, 36(3), 340-348. doi:10.1097/ 00004583-199703000-00012

Böttche, M., Kuwert, P., \& Knaevelsrud, C. (2012). Posttraumatic stress disorder in older adults: An overview of characteristics and treatment approaches. International Journal of Geriatric Psychiatry, 27(3), 230-239. doi:10.1002/gps.2725

Boudoukha, A. H., Przygodzki-Lionet, N., \& Hautekeete, M. (2016). Traumatic events and early maladaptive schemas (EMS): Prison guard psychological vulnerability. Revue Européenne de Psychologie Appliquée. Revue Européenne de Psychologie Appliquéel European Review of Applied Psychology, 66(4), 181-187. doi:10.1016/j.erap.2011.05.004

Classen, C. C., Palesh, O. G., \& Aggarwal, R. (2005). Sexual revictimization: A review of the empirical literature. Trauma, Violence, \& Abuse, 6(2), 103-129. doi:10.1177/ 1524838005275087

Cloitre, M., Courtois, C. A., Charuvastra, A., Carapezza, R., Stolbach, B. C., \& Green, B. L. (2011). Treatment of complex PTSD: Results of the ISTSS expert clinician survey on best practices. Journal of Traumatic Stress, 24(6), 615-627. doi:10.1002/jts.20697

Cloitre, M., Garvert, D. W., Brewin, C. R., Bryant, R. A., \& Maercker, A. (2013). Evidence for proposed ICD-11 PTSD and complex PTSD: A latent profile analysis. European Journal of Psychotraumatology, 4(1), 20706. doi:10.3402/ejpt.v4i0.20706

Cloitre, M., Shevlin, M., Brewin, C. R., Bisson, J. I., Roberts, N. P., Maercker, A., ... Hyland, P. (2018). The International Trauma Questionnaire: Development of a selfreport measure of ICD-11 PTSD and complex PTSD. Acta Psychiatrica Scandinavica, 138(6), 536-546. doi:10.1111/acps.12956

Cockram, D. M., Drummond, P. D., \& Lee, C. W. (2010). Role and treatment of early maladaptive schemas in Vietnam veterans with PTSD. Clinical Psychology \& Psychotherapy: An International Journal of Theory \& Practice, 17(3), 165-182. doi:10.1002/cpp.690

Creech, S. K., Benzer, J. K., Liebsack, B. K., Proctor, S., \& Taft, C. T. (2013). Impact of coping style and PTSD on family functioning after deployment in operation desert shield/ storm returnees. Journal of Traumatic Stress, 26(4), 507-511. doi:10.1002/jts.21823

Dall, T. M., Gallo, P. D., Chakrabarti, R., West, T., Semilla, A. P., \& Storm, M. V. (2013). An aging population and growing disease burden will require alarge and specialized health care workforce by 2025. Health Affairs, 32(11), 2013-2020. doi:10.1377/hlthaff. 2013.0714

Dutra, L., Callahan, K., Forman, E., Mendelsohn, M., \& Herman, J. (2008). Core schemas and suicidality in a chronically traumatized population. The Journal of nervous and mental disease, 196(1), 71-74. doi:10.1097/NMD.0b013e31815fa4c1

Edworthy, Z., Chasey, R., \& Williams, H. (2008). The role of schema and appraisals in the development of post-traumatic stress symptoms following birth. Journal of Reproductive and Infant Psychology, 26(2), 123-138. doi:10.1080/02646830801918422

Ehlers, A., \& Clark, D. M. (2000). A cognitive model of posttraumatic stress disorder. Behaviour Research and Therapy, 38(4), 319-345. doi:10.1016/S0005-7967(99)00123-0

Elklit, A., Hyland, P., \& Shevlin, M. (2014). Evidence of symptom profiles consistent with posttraumatic stress disorder and complex posttraumatic stress disorder in different 
trauma samples. European Journal of Psychotraumatology, 5(1), 24221. doi:10.3402/ejpt. v5.24221

Estévez, A., Jauregui, P., Ozerinjauregi, N., \& Herrero-Fernández, D. (2017). The role of early maladaptive schemas in the appearance of psychological symptomatology in adult women victims of child abuse. Journal of Child Sexual Abuse, 26(8), 889-909. doi:10. 1080/10538712.2017.1365318

Floyd, M., Rice, J., \& Black, S. R. (2002). Recurrence of posttraumatic stress disorder in late life: A cognitive aging perspective. Journal of Clinical Geropsychology, 8(4), 303-311. doi: Doi.org/10.1023/A:1019679307628

Foa, E. B., Ehlers, A., Clark, D. M., Tolin, D. F., \& Orsillo, S. M. (1999). The posttraumatic cognitions inventory (PTCI): Development and validation. Psychological Assessment, 11(3), 303. doi:10.1037//1040-3590.11.3.303

Fritz, M. S., \& MacKinnon, D. P. (2007). Required sample size to detect the mediated effect. Psychological Science, 18(3), 233-239. doi:10.1111/j.1467-9280.2007.01882.x

Gay, L. E., Harding, H. G., Jackson, J. L., Burns, E. E., \& Baker, B. D. (2013). Attachment style and early maladaptive schemas as mediators of the relationship between childhood emotional abuse and intimate partner violence. Journal of Aggression, Maltreatment \& Trauma, 22(4), 408-424. doi:10.1080/10926771.2013.775982

Goenjian, A. K., Najarian, L. M., Pynoos, R. S., Steinberg, A. M., Manoukian, G., Tavosian, A., \& Fairbanks, L. A. (1994). Posttraumatic stress disorder in elderly and younger adults after the 1988 earthquake in Armenia. Am J Psychiatry, 151(6), 895-901. doi:10.1176/ajp. 151.6.895

Grenade, L., \& Boldy, D. (2008). Social isolation and loneliness among older people: Issues and future challenges in community and residential settings. Australian Health Review, 32(3), 468-478. doi:10.1071/AH080468

Harding, H. G., Burns, E. E., \& Jackson, J. L. (2012). Identification of child sexual abuse survivor subgroups based on early maladaptive schemas: Implications for understanding differences in posttraumatic stress disorder symptom severity. Cognitive Therapy and Research, 36(5), 560-575. doi:10.1007/s10608-011-9385-8

Hayes, A. F. (2013). Introduction to mediation, moderation, and conditional process analysis: A regression-based approach. London, UK: Guilford Press.

Hiskey, S., Luckie, M., Davies, S., \& Brewin, C. R. (2008). The phenomenology of reactivated trauma memories in older adults: A preliminary study. Aging \& Mental Health, 12(4), 494-498. doi:10.1080/13607860802224367

Hoffart, A., Sexton, H., Hedley, L. M., Wang, C. E., Holthe, H., Haugum, J. A., ... Holte, A. (2005). The structure of maladaptive schemas: A confirmatory factor analysis and a psychometric evaluation of factor-derived scales. Cognitive Therapy and Research, 29(6), 627-644. doi:10.1007/s10608-005-9630-0

Hyland, P., Shevlin, M., Elklit, A., Murphy, J., Vallières, F., Garvert, D. W., \& Cloitre, M. (2017). An assessment of the construct validity of the ICD-11 proposal for complex posttraumatic stress disorder. Psychological Trauma: Theory, Research, Practice, and Policy, 9(1), 1. doi:10.1037/tra0000114]

Karatzias, T., Jowett, S., Begley, A., \& Deas, S. (2016). Early maladaptive schemas in adult survivors of interpersonal trauma: Foundations for a cognitive theory of psychopathology. European Journal of Psychotraumatology, 7(1), 30713. doi:10.3402/ejpt.v7.30713

Karatzias, T., Cloitre, M., Maercker, A., Kazlauskas, E., Shevlin, M., Hyland, P., ... Brewin, C. R. (2017a). PTSD and Complex PTSD: ICD-11 updates on concept and measurement in the UK, USA, Germany and Lithuania. European Journal of Psychotraumatology, 8(sup7), 1418103-1418103. doi:10.1080/20008198 
Karatzias, T., Shevlin, M., Fyvie, C., Hyland, P., Efthymiadou, E., Wilson, D., ... Cloitre, M. (2017b). Evidence of distinct profiles of Posttraumatic Stress Disorder (PTSD) and Complex Posttraumatic Stress Disorder (CPTSD) based on the new ICD-11 Trauma Questionnaire (ICD-TQ). Journal of Affective Disorders, 207, 181-187. doi:10.1016/j.jad. 2016.09.032

Karatzias, T., Hyland, P., Bradley, A., Cloitre, M., Roberts, N. P., Bisson, J. I., \& Shevlin, M. (2019a). Risk factors and comorbidity of ICD-11 PTSD and complex PTSD: Findings from a trauma-exposed population based sample of adults in the United Kingdom. Depression and Anxiety, 1-8. doi:10.1002/da.22934

Karatzias, T., Murphy, P., Cloitre, M., Bisson, J., Roberts, N., Shevlin, M., ... MasonRoberts, S. (2019b). Psychological interventions for ICD-11 complex PTSD symptoms: Systematic review and meta-analysis. Psychological Medicine, 49(11), 1761-1775. doi:10. 1017/S0033291719000436

Kindynis, S., Burlacu, S., Louville, P., \& Limosin, F. (2013). Effect of schema-focused therapy on depression, anxiety and maladaptive cognitive schemas in the elderly. L'Encephale, 39(6), 393-400. doi:10.1016/j.encep.2013.04.002

Lecigne, M., \& Tapia, G. (2018). The mediating role of early maladaptive schemas on relations between post-traumatic stress disorder symptom severity and illicit substance use disorder. Journal of Substance Use, 23(6), 634-639. doi:10.1080/14659891.2018.1489005

Lumley, M. N., \& Harkness, K. L. (2007). Specificity in the relations among childhood adversity, early maladaptive schemas, and symptom profiles in adolescent depression. Cognitive Therapy and Research, 31(5), 639-657. doi:10.1007/s10608-006-9100-3

MacDonald, K., Thomas, M. L., Sciolla, A. F., Schneider, B., Pappas, K., Bleijenberg, G., ... Wingenfeld, K. (2016). Minimization of childhood maltreatment is common and consequential: Results from a large, multinational sample using the childhood trauma questionnaire. PLoS One, 11(1), e0146058-e0146058. doi:10.1371/journal.pone.0146058

Maercker, A., Brewin, C. R., Bryant, R. A., Cloitre, M., Reed, G. M., van Ommeren, M., ... Saxena, S. (2013). Proposals for mental disorders specifically associated with stress in the International Classification of Diseases-11. The Lancet, 381(9878), 1683-1685. doi:10. 1016/S0140-6736(12)62191-6

Neupert, S. D., Almeida, D. M., \& Charles, S. T. (2007). Age differences in reactivity to daily stressors: The role of personal control. The Journals of Gerontology Series B: Psychological Sciences and Social Sciences, 62(4), P216-P225. doi:10.1093/geronb/62.4. P216

Parker, G., Lie, D., Siskind, D. J., Martin-Khan, M., Raphael, B., Crompton, D., \& Kisely, S. (2016). Mental health implications for older adults after natural disasters-a systematic review and meta-analysis. International Psychogeriatrics, 28(1), 11-20. doi:10.1017/ S1041610215001210

Pauwels, E., Claes, L., Dierckx, E., Debast, I., Van Alphen, S. P. J. (B. )., Rossi, G., ... Peuskens, H. (2014). Age neutrality of the young schema questionnaire in patients with a substance use disorder. International Psychogeriatrics, 26(8), 1317-1326. doi:10.1017/ S1041610214000519

Preacher, K. J., \& Hayes, A. F. (2004). SPSS and SAS procedures for estimating indirect effects in simple mediation models. Behavior Research Methods, 36(4), 717-731. doi:10. 3758/BF03206553

Preacher, K. J., \& Kelley, K. (2011). Effect size measures for mediation models: Quantitative strategies for communicating indirect effects. Psychological Methods, 16(2), 93. doi:10.1037/a0022658 
Price, J. P. (2007). Cognitive schemas, defence mechanisms and post-traumatic stress symptomatology. Psychology and Psychotherapy: Theory, Research and Practice, 80(3), 343-353. doi:10.1348/147608306X144178

Pynoos, R. S., Steinberg, A. M., \& Wraith, R. (1995). A developmental model of childhood traumatic stress. In D. Cicchetti \& D. J. Cohen (Eds.), Wiley series on personality processes. Developmental psychopathology, Vol. 2. Risk, disorder, and adaptation (pp. 72-95). Oxford, UK: John Wiley \& Sons.

Rezaei, M., Ghazanfari, F., \& Rezaee, F. (2016). The role of childhood trauma, early maladaptive schemas, emotional schemas and experimental avoidance on depression: A structural equation modeling. Psychiatry Research, 246, 407-414. doi:10.1016/j.psychres. 2016.10.037

Roemmele, M., \& Messman-Moore, T. L. (2011). Child abuse, early maladaptive schemas, and risky sexual behavior in college women. Journal of Child Sexual Abuse, 20(3), 264-283. doi:10.1080/10538712.2011.575445

Ruzich, M. J., Looi, J. C. L., \& Robertson, M. D. (2005). Delayed onset of posttraumatic stress disorder among male combat veterans: A case series. The American Journal of Geriatric Psychiatry, 13(5), 424-427. doi:10.1097/00019442-200505000-00013

Scher, C. D., Stein, M. B., Asmundson, G. J., McCreary, D. R., \& Forde, D. R. (2001). The childhood trauma questionnaire in a community sample: Psychometric properties and normative data. Journal of Traumatic Stress, 14(4), 843-857. doi:10.1023/ A:1013058625719

Thomson, P., \& Jaque, S. V. (2019). History of childhood adversity and coping strategies: Positive flow and creative experiences. Child Abuse \& Neglect, 90, 185-192. doi:10.1016/j. chiabu.2018.12.019

Vasilopoulou, E., Michalska da Rocha, B., Forsyth, K., Guzman, A., \& Karatzias, T. (2019). Factors associated with the severity of Post-Traumatic Stress Disorder and Complex Post-Traumatic Stress Disorder in older adults: A systematic review. Unpublished Manuscript, University of Edinburgh.

Videler, A., van Royen, R. R., \& van Alphen, S. B. (2012). Schema therapy with older adults: Call for evidence. International Psychogeriatrics, 24(7), 1186-1187. doi:10.1017/ S1041610211002638

World Health Organisation (2018). International classification of diseases 11th revision. Retrieved from https://icd.who.int/ct11_2018/icd11_mms/en/release\#/

Wright, M. O. D., Crawford, E., \& Del Castillo, D. (2009). Childhood emotional maltreatment and later psychological distress among college students: The mediating role of maladaptive schemas. Child Abuse \& Neglect, 33(1), 59-68. doi:10.1016/j.chiabu.2008.12.007

Young, J. E., Klosko, J. S., \& Weishaar, M. E. (2003). Schema therapy: A practitioner's guide. New York, NY/London, UK: Guilford Press.

Young, J. E. (2014). Young schema questionnaire-3. New York, NY: Cognitive Therapy Center. 